\title{
Keratoprosthesis, silicone oil placement, and fluocinolone acetonide implant for treatment of uveitis-associated hypotony and keratopathy
}

\author{
Arman Mosenia ${ }^{1,2}$, Miel Sundararajan², Jay M. Stewart ${ }^{2}$ and Julie M. Schallhorn²*
}

\begin{abstract}
Purpose: To describe a case series of combined Boston Type 1 Keratoprosthesis with pars plana vitrectomy, silicone oil placement, and fluocinolone acetonide intravitreal $0.59 \mathrm{mg}$ implant (RETISERT ${ }^{\oplus}$ ), and report its safety and efficacy in preventing phthisis bulbi in patients with uveitis-associated hypotony and concurrent corneal edema.

Findings: A retrospective review of patients with chronic uveitis, corneal decompensation and concurrent hypotony who underwent the combined approach described here between 2015 and 2020 was conducted. Three patients were treated using the combined approach. Post-operative recovery was unremarkable in all cases and the patients' corneal condition remained stable on follow up. No patient developed phthisis, retroprosthetic membrane, or infectious endophthalmitis. Average intraocular pressure one year after intervention was 2.7 to $6.4 \mathrm{mmHg}$ higher compared to a year prior.
\end{abstract}

Conclusions: The approach described is potentially safe and effective in preventing phthisis and membrane formation in uveitis-associated hypotony and keratopathy.

\section{Introduction}

Hypotony associated with uveitis is an end-stage manifestation of severe ocular inflammation and is associated with loss of visual acuity. It emerges when aqueous production by the ciliary body does not match the outflow [1], and can be present in up to $8 \%$ of cases of uveitis [2]. This condition is particularly challenging to treat, and in conjunction with persistent inflammation, can result in phthisis bulbi. Currently, there are no definite treatments for hypotony; however, multiple treatment options have been proposed, including intravitreal injection of viscoelastic material, intraocular gas or silicone oil, topical dopaminergic agonist administration

\footnotetext{
* Correspondence: Julie.Schallhorn@ucsf.edu

${ }^{2}$ Department of Ophthalmology, University of California San Francisco, 490 Illinois Street, San Francisco, CA 94158, USA

Full list of author information is available at the end of the article
}

(ibopamine), and pars plana vitrectomy with ciliary membrane removal [3-8].

Long standing hypotony may also cause corneal endothelial pump dysfunction and stromal edema [9]. While the pathophysiology of corneal endothelial decompensation within this context is not well understood, alterations in the composition and flow of aqueous are believed to lead to poor tissue oxygenation and breakdown of the blood-aqueous barrier [10]. Corneal transplantation in the setting of hypotony is technically challenging, and recurrence of edema is common. Consequently, patients often receive multiple corneal transplantations without resolution. Boston Type 1 Keratoprosthesis combined with silicone oil for treatment of concurrent hypotony and corneal edema has shown to improve vision [11]. However, a common complication of this approach is the formation of retroprosthetic membrane [11]. 
The intravitreal $0.59 \mathrm{mg}$ fluocinolone implant (RETISERT $^{\oplus}$, Bausch \& Lomb, Rochester, NY), is a well described treatment for uveitis, providing excellent control of inflammation for 24 to 30 months after implantation [12]. It exerts its effect locally, and may spare the side effects of systemic immunosuppression in some patients [13]. Although it has been associated with a significant risk of developing ocular hypertension and glaucoma [14], it has not been found to improve intraocular pressure when implanted in hypotonus eyes [15].

The purpose of this study is to report the authors' experience in treating corneal edema in the setting of uveitis-associated hypotony with combined Boston Type 1 Keratoprosthesis placement (Massachusetts Eye and Ear, Boston, MA), silicone oil injection, and $0.59 \mathrm{mg}$ fluocinolone acetonide intravitreal implant.

\section{Materials and methods}

This is a retrospective study of patients with hypotony who underwent fluocinolone acetonide implantation and single-stage keratoprosthesis with silicone oil injection between 2015 and 2020. Patients with chronic uveitis who had evidence of corneal decompensation with concurrent hypotony were included, and their electronic health records were reviewed.All patients underwent surgery for an indication of corneal edema in the setting of uveitis and hypotony, and all patients elected to undergo fluocinolone acetonide implantation for uveitis control. All patients underwent 23-gauge pars plana vitrectomy with silicone oil injection $(5000 \mathrm{cSt})$. One patient had a pre-existing retinal detachment. Intraocular pressures after keratoprosthesis placement were estimated by scleral pressure and were measured using pneumatonometry in the inferotemporal quadrant to compare with corneal pressure measurements prior to keratoprosthesis placement [16].

\section{Case presentations \\ Case 1}

A 47-year-old woman with a history of chronic unilateral non-granulomatous anterior uveitis with cystoid macular edema (CME) of the left eye presented with eye pain for surgical visual rehabilitation evaluation (Table 1). Her history was complicated by non-clearing vitreous hemorrhage due to neovascularization of the pars plana, and pseudophakic bullous keratopathy. She had previously undergone Descemet's stripping endothelial keratoplasty (DSAEK) followed by penetrating keratoplasty (PK), both of which were complicated by graft failure. Therefore, she was treated with pars plana vitrectomy, silicone oil placement, and fluocinolone acetonide intravitreal implant insertion with repeat PK. Six months after this surgery, she underwent silicone oil removal, but developed hypotony with choroidal detachment and an overlying retinal detachment. Due to persistently low intraocular pressure, a combined aphakic keratoprosthesis with retinal detachment repair and silicone oil injection was carried out. Visual acuity prior to surgery was light perception (LP) and intraocular pressure was 4 $\mathrm{mmHg}$. The retinal detachment was noted to be complex, and the vision became no light perception (NLP) after repair. The retina has remained attached at oneyear follow up and the patient has maintained a stable keratoprosthesis without bandage contact lens placement.

\section{Case 2}

A 53-year-old woman with bilateral steroid response glaucoma and chronic panuveitis presented for surgical treatment of corneal edema and hypotony (Table 1 ). Her left eye was noted to be hypotonus with diffuse corneal edema. Visual acuity in the left eye was light perception (LP), and intraocular pressure was $1 \mathrm{mmHg}$ at the time of initial evaluation. She was offered a single stage combined fluocinolone acetonide implantation with an aphakic keratoprosthesis and silicone oil insertion for visual rehabilitation. The operation was completed without complication, with a resultant BCVA of 20/800 and an intraocular pressure of $13 \mathrm{mmHg}$. Bandage contact lens fitting proved difficult due to the unusual ocular surface contour, but the ocular surface has remained stable without a bandage contact lens placement. The patient has not developed a retroprosthetic membrane at one-year follow up.

\section{Case 3}

A 57-year-old man with a history of bilateral immune reconstitution panuveitis secondary to CMV retinitis in the setting of human immunodeficiency virus (HIV) developed significant hypotony in the right eye requiring repeat injections of hyaluronic acid (Table 1). The left eye had no light perception. Vision in the affected eye

Table 1 Clinical profile of patients

\begin{tabular}{|c|c|c|c|c|c|c|c|c|}
\hline Case & $\begin{array}{l}\text { Age/ } \\
\text { Sex }\end{array}$ & $\begin{array}{l}\text { Uveitis } \\
\text { class }\end{array}$ & Chief complaint & Previous Transplants & Vision, Before & $\begin{array}{l}\text { Vision, } \\
\text { After }\end{array}$ & Complications & Follow Up (years) \\
\hline 1 & $47 / F$ & Anterior, unilateral & Pain, visual rehabilitation & 3 & $\mathrm{LP}$ & NLP & None & 1 \\
\hline 2 & $53 / F$ & Panuveitis, bilateral & Corneal edema & 0 & LP & $20 / 800$ & None & 1 \\
\hline 3 & $57 / M$ & Panuveitis, bilateral & Declining vision & 2 & $\mathrm{HM}$ & $20 / 125$ & None & 5.5 \\
\hline
\end{tabular}

LP Light perception, NLP No light perception, HM Hand motion 
was hand motions. He had previously undergone three vitrectomies and two keratoplasties. The keratoplasties had presumably failed due to hypotony. He developed an inferior retinal detachment, and a combined surgical approach was pursued for aphakic keratoprosthesis placement, removal of existing intraocular lens, pars plana vitrectomy and silicone oil injection. He had a fluocinolone acetonide implant, which was most recently exchange a year prior to the surgery. Post-operative recovery was unremarkable. At one-year follow up, best corrected visual acuity was 20/125. He has subsequently undergone multiple dexamethasone intravitreal steroid implant (OZURDEX ${ }^{\oplus}$, Allergan, Irvine, CA) injections and four supplemental intravitreal silicone oil injections to increase the intraocular pressure.

He has been maintained without bandage contact lens placement, as achieving an appropriate fit was difficult. The ocular surface was stable, and he did not have a retroprosthetic membrane for 5 years after surgery. However, he developed a small epithelial defect at 5 years that responded within 1 week to amniotic membrane placement.

\section{Discussion}

Hypotony may lead into several irreversible, visionimpairing complications in the posterior segment. The resulting shrinkage and collapse of the scleral wall due to low intraocular pressure (IOP) is thought to fold and wrinkle the chorio-retina, particularly in the peri-foveal region, distorting neurosensory elements with permanent visual loss [1]. Additionally, the malformation of the globe may change retinal capillary permeability, resulting in cystoid macular edema as seen in all three cases here. Therefore, early detection of hypotony and its management dictates final visual outcomes in these patients. In all three cases, the average pressure in the affected eye was higher after keratoprosthesis insertion compared to a year prior (Table 2). In one of three, silicone oil was injected at least once in the year prior to surgery. While average IOP one month prior to and following silicone oil insertion were nearly identical at $6 \mathrm{mmHg}(n=2)$ and $6.7 \mathrm{mmHg}(n=3)$, respectively, average IOP before and after silicone oil placement with keratoprosthesis was 4 $\mathrm{mmHg}(n=1)$ and $10 \mathrm{mmHg}(n=3)$. These findings

Table 2 Average IOP within one year before and after combined keratoprosthesis and silicone oil insertion

\begin{tabular}{llll}
\hline Case & Pre-Intervention IOP & Post-Intervention IOP & $P$-value $<\mathbf{0 . 0 5}$ \\
\hline 1 & $6.07[4.797 .35]$ & $12.50[10.32 ~ 14.68]$ & $*$ \\
2 & $8.70[3.7413 .66]$ & $11.40[10.4712 .33]$ & \\
3 & $6.93[5.937 .95]$ & $10.45[7.4913 .43]$ & $*$ \\
\hline
\end{tabular}

Numbers in brackets are confidence intervals of the mean pressures. All pressured reported in $\mathrm{mmHg}$. $P$ value corresponds to the difference between pre-intervention and post-intervention mean pressures suggest that combined keratoprosthesis insertion and silicone oil placement may help maintain higher IOP when compared to silicone oil alone, thus lowering the probability of hypotony maculopathy.

In phthisical eyes, the corneal epithelium is often nor$\mathrm{mal}$, and the ensuing edema is stromal due to endothelial decompensation [9]. This has been shown to be reversible by addressing the underlying cause of hypotony, in trabeculectomy for example [17]. Nevertheless, uveitis-induced hypotony is challenging to treat and often persists. Therefore, the main treatment option is keratoplasty (DSAEK or PK), which has a dim chance of success without addressing the underlying cause and improving IOP. Two of the three cases reported here had received corneal transplants, all of which had failed. Therefore, permanent keratoprosthesis may improve vision and help minimize the number of surgical interventions, especially in those with recurring graft failure.

Retroprosthetic membrane formation is a common occurrence after keratoprosthesis surgery [18]. In a prior study describing combined keratoprosthesis surgery and silicone oil insertion for treatment of hypotony secondary to multiple etiologies, retroprosthetic membrane was seen in $54 \%$ of patients [11]. In those with uveitis, however, this can be more prevalent due to increased inflammation, affecting visual acuity and predisposing them to sterile keratolysis [19]. Notably, none of the cases reported here developed retroprosthetic membranes. The presence of the intravitreal fluocinolone implant likely contributed to this, as local high dose steroids have been associated with decreased vascular permeability and fibroblast proliferation, and have been shown to lengthen the time to retroprosthetic membrane development [18]. Specifically, the fluocinolone acetonide implant has been associated with improved long-term outcomes with glaucoma drainage device placement [20].

All eyes in this study also had stable ocular surfaces without the need for a bandage contact lens. The patients in this study had difficulty getting a lens fit owing to the abnormal contour of their ocular surface in the setting of a pre-phthisical state prior to keratoprosthesis placement. Even extremely flat, large diameter lenses were unable to be retained successfully. Despite this, these patients did not experience epithelial breakdown and keratolysis. Stability of the ocular surface in the setting of silicone oil and keratoprosthesis placement to treat hypotony has previously been noted [11, 21]. We theorize that shrinkage of the eye due to the prephthisical state prior to surgery affords better protection of the ocular surface from the eyelids, and thus enables more stability of the epithelium.

This study has several strengths, including unremarkable post-operative recovery and absence of any 
complications, particularly retroprosthetic membrane formation, infectious endophthalmitis and sterile keratolysis in uveitis-associated hypotony and keratopathy. While inferotemporal scleral pneumatonometry is a promising method for measuring IOP in those with keratoprosthesis, this has not been validated in eyes with hypotony [16]. Future work can focus on further characterizing the impact of this intervention on IOP. Regardless, the combined approach was clinically effective in preventing phthisis for at least one year after surgery in these patients.

\section{Conclusion}

Combined treatment of uveitis-associated hypotony and keratopathy with Boston Type 1 Keratoprosthesis, silicone oil placement, and fluocinolone acetonide implant may be a safe and effective method to prevent phthisis.

\section{Abbreviations \\ IOP: Intraocular pressure; LP: Light perception; NLP: No light perception; HM: Hand motion}

\section{Acknowledgements}

None.

\section{Authors' contributions}

JMSC, JMSt, and MS performed the surgeries and contributed to patient management. JMSC and AM jointly designed the study and created the initial draft. All authors discussed the results, contributed to manuscript revisions, and approved the final manuscript.

\section{Funding}

This research did not receive any specific grants from funding agencies in the public, commercial, or not-for-profit sectors.

\section{Availability of data and materials}

The data used and analyzed during the current study are available from the corresponding author on reasonable request.

\section{Declarations}

\section{Ethics approval and consent to participate}

This study was reviewed and approved by the University of California, San Francisco Institutional Review Board, and conformed to the tenets of the Declaration of Helsinki.

\section{Consent for publication}

Not applicable. Patients are entirely unidentifiable, and no images are included.

\section{Competing interests}

The authors report no competing interests.

\author{
Author details \\ ${ }^{1}$ School of Medicine, University of California San Francisco, 533 Parnassus \\ Ave, San Francisco, CA 94143, USA. ²Department of Ophthalmology, \\ University of California San Francisco, 490 Illinois Street, San Francisco, CA \\ 94158, USA.
}

Received: 13 December 2021 Accepted: 15 January 2022 Published online: 04 February 2022

\section{References}

1. Costa VP, Arcieri ES (2007) Hypotony maculopathy. Acta Ophthalmol Scand 85(6):586-597. https://doi.org/10.1111/j.1600-0420.2007.00910.x
2. Sen HN, Drye LT, Goldstein DA, Larson TA, Merrill PT, Pavan PR, Sheppard JD, Burke A, Srivastava SK, Jabs DA, for the Multicenter Uveitis Steroid Treatment (MUST) Trial Research Group (2012) Hypotony in patients with uveitis: the multicenter uveitis steroid treatment (MUST) trial. Ocul Immunol Inflamm 20(2):104-112. https://doi.org/10.3109/09273948.2011.647228

3. Ugahary LC, Ganteris E, Veckeneer M, Cohen AC, Jansen J, Mulder PGH, van Meurs JC (2006) Topical ibopamine in the treatment of chronic ocular hypotony attributable to vitreoretinal surgery, uveitis, or penetrating trauma. Am J Ophthalmol 141(3):571-573. https://doi.org/10.1016/j.ajo.2005.09.034

4. O'Connell SR, Majji AB, Humayun MS, de Juan E (2000) The surgical management of hypotony. Ophthalmology 107(2):318-323. https://doi.org/1 0.1016/s0161-6420(99)00047-0

5. Yu EN, Paredes I, Foster CS (2007) Surgery for hypotony in patients with juvenile idiopathic arthritis-associated uveitis. Ocul Immunol Inflamm 15(1): 11-17. https://doi.org/10.1080/09273940601147729

6. Kapur R, Birnbaum AD, Goldstein DA, Tessler HH, Shapiro MJ, Ulanski LJ, Blair MP (2010) Treating uveitis-associated hypotony with pars plana vitrectomy and silicone oil injection. Retina Phila Pa 30(1):140-145. https:// doi.org/10.1097//AE.0b013e3181b32f06

7. Tosi GM, Schiff W, Barile G, Yoshida N, Chang S (2005) Management of severe hypotony with intravitreal injection of viscoelastic. Am J Ophthalmol 140(5):952-954. https://doi.org/10.1016/j.ajo.2005.06.019

8. Cadera W, Harding PW, Gonder JR, Hooper PL (1993) Management of severe hypotony with intravitreal injection of Healon. Can J Ophthalmol J Can Ophtalmol 28:236-237

9. Hatton MP, Perez VL, Dohlman CH (2004) Corneal oedema in ocular hypotony. Exp Eye Res 78(3):549-552. https://doi.org/10.1016/j.exer.2003.06. 003

10. Schubert HD (1996) Postsurgical hypotony: relationship to fistulization, inflammation, chorioretinal lesions, and the vitreous. Surv Ophthalmol 41(2): 97-125. https://doi.org/10.1016/S0039-6257(96)80001-4

11. Chan CC, Holland EJ, Sawyer WI, Neff KD, Petersen MR, Riemann CD (2011) Boston type 1 keratoprosthesis combined with silicone oil for treatment of hypotony in prephthisical eyes. Cornea 30(10):1105-1109. https://doi.org/1 0.1097/ICO.0b013e318207f3bb

12. The Multicenter Uveitis Steroid Treatment (MUST) Trial Follow-up Study Research Group (2015) Quality of life and risks associated with systemic anti-inflammatory therapy versus Fluocinolone Acetonide intraocular implant for intermediate uveitis, posterior uveitis, or Panuveitis. Ophthalmology 122(10):1976-1986. https://doi.org/10.1016/j.ophtha.2015.06. 043

13. The Multicenter Uveitis Steroid Treatment (MUST) Trial Follow-up Study Research Group (2015) Benefits of systemic anti-inflammatory therapy versus Fluocinolone Acetonide intraocular implant for intermediate uveitis, posterior uveitis, and Panuveitis. Ophthalmology 122(10):1967-1975. https:// doi.org/10.1016/j.ophtha.2015.06.042

14. Friedman DS, Holbrook JT, Ansari H, Alexander J, Burke A, Reed SB, Katz J, Thorne JE, Lightman SL, Kempen JH (2013) Risk of elevated intraocular pressure and Glaucoma in patients with uveitis. Ophthalmology 120(8): 1571-1579. https://doi.org/10.1016/j.ophtha.2013.01.025

15. Hebson C, Martin D, Srivastava S (2009) Intraocular pressure response in patients with Hypotony and uveitis after intravitreal Fluocinolone Acetonide implantation (RetisertTM). Invest Ophthalmol Vis Sci 50:3574-3574

16. Lin CC, Chen A, Jeng BH, Porco TC, Ou Y, Han Y (2014) Scleral intraocular pressure measurement in cadaver eyes pre- and postkeratoprosthesis implantation. Invest Ophthalmol Vis Sci 55(4):2244-2250. https://doi.org/1 $0.1167 /$ iovs.13-13153

17. Sriram A, Tania Tai TY (2017) Resolution of chronic corneal edema after surgical treatment for ocular Hypotony. J Glaucoma 26(6):e187-e189. https://doi.org/10.1097/IJG.0000000000000644

18. Rudnisky CJ, Belin MW, Todani A, Al-Arfaj K, Ament JD, Zerbe BJ, Ciolino JB, Boston Type 1 Keratoprosthesis Study Group (2012) Risk factors for the development of retroprosthetic membranes with Boston keratoprosthesis type 1: multicenter study results. Ophthalmology 119(5):951-955. https://doi. org/10.1016/j.ophtha.2011.11.030

19. Sivaraman KR, Hou JH, Allemann N, de la Cruz J, Cortina MS (2013) Retroprosthetic membrane and risk of sterile keratolysis in patients with type I Boston Keratoprosthesis. Am J Ophthalmol 155(5):814-822. https:// doi.org/10.1016/j.ajo.2012.11.019

20. Hennein L, Hou J, Stewart JM, Lowry EA, Jiang Z, Enanoria WTA, Han Y (2016) Comparison of surgical outcome after Ahmed valve implantation for 
patients with and without Fluocinolone intravitreal implant (Retisert). J

Glaucoma 25(9):e772-e776. https://doi.org/10.1097/IJG.0000000000000497

21. Iyer G, Srinivasan B, Gupta J, Rishi P, Sen PR, Bhende P, Gopal L,

Padmanabhan P (2011) Boston keratoprosthesis for keratopathy in eyes with

retained silicone oil: a new indication. Cornea 30(10):1083-1087. https://doi. org/10.1097//CO.0b013e318213a8b5

\section{Publisher's Note}

Springer Nature remains neutral with regard to jurisdictional claims in published maps and institutional affiliations.

Submit your manuscript to a SpringerOpen ${ }^{\circ}$ journal and benefit from:

- Convenient online submission

- Rigorous peer review

- Open access: articles freely available online

- High visibility within the field

- Retaining the copyright to your article

Submit your next manuscript at $\boldsymbol{\wedge}$ springeropen.com 